THEORY OF COMPUTING

\title{
On a Segment Partition for Entropy Estimation
}

E. A. Timofeev ${ }^{1}$

DOI: $10.18255 / 1818-1015-2020-1-40-47$

${ }^{1}$ P. G. Demidov Yaroslavl State University, 14 Sovetskaya, Yaroslavl 150003, Russia.

MSC2020: 94A17

Research article

Full text in Russian
Received November 23, 2019

After revision February 18, 2020

Accepted February 28, 2020

Let $Q_{n}$ be a partition of the interval $[0,1]$ defines as

$$
\begin{aligned}
& Q_{1}=\left\{0, q^{2}, q, 1\right\} . \\
& Q_{n+1}^{\prime}=q Q_{n} \cap q^{2} Q_{n}, \quad Q_{n+1}^{\prime \prime}=q^{2}+q Q_{n} \cap q Q_{n}, \quad Q_{n+1}^{\prime \prime \prime}=q^{2}+q Q_{n} \cap q+q^{2} Q_{n}, \\
& Q_{n+1}=Q_{n+1}^{\prime} \cup Q_{n+1}^{\prime \prime} \cup Q_{n+1}^{\prime \prime},
\end{aligned}
$$

where $q^{2}+q=1$

The sequence $d=1,2,1,0,1,2,1,0,1,0,1,2,1,0,1,2,1, \ldots$ defines as follows.

$$
\begin{aligned}
& d_{1}=1, d_{2}=2, d_{4}=0 ; \\
& d\left[2 F_{2 n}+1: 2 F_{2 n+1}+1\right]=d\left[1: 2 F_{2 n-1}+1\right] \\
& \quad n=0,1,2, \ldots ; \\
& d\left[2 F_{2 n+1}+2: 2 F_{2 n+1}+2 F_{2 n-2}\right]=d\left[2 F_{2 n-1}+2: 2 F_{2 n}\right] \\
& d\left[2 F_{2 n+1}+2 F_{2 n-2}+1: 2 F_{2 n+1}+2 F_{2 n-1}+1\right]=d\left[1: 2 F_{2 n-3}+1\right] \\
& d\left[2 F_{2 n+1}+2 F_{2 n-1}+2: 2 F_{2 n+2}\right]=d\left[2 F_{2 n-1}+2: 2 F_{2 n}\right] \\
& \quad n=1,2,3, \ldots ;
\end{aligned}
$$

where $F_{n}$ are Fibonacci numbers $\left(F_{-1}=0, F_{0}=F_{1}=1\right)$.

The main result of this paper.

Theorem.

$$
\begin{gathered}
Q_{n}^{\prime}=1-Q_{n}^{\prime \prime \prime}=\left\{\sum_{i=1}^{k} q^{n+d_{i}}, k=0,1, \ldots, m_{n}\right\}, \\
Q_{n}^{\prime \prime}=1-Q_{n}^{\prime \prime}=\left\{q^{2}+\sum_{i=m_{n}}^{k} q^{n+d_{i}}, k=m_{n}-1, m_{n}, \ldots, m_{n+1}\right\},
\end{gathered}
$$

where $m_{2 n}=2 F_{2 n-2}, m_{2 n+1}=2 F_{2 n-1}+1$.

Keywords: measure; metric; entropy; estimation; unbiased; self-similarity; Bernoulli measure

\section{INFORMATION ABOUT THE AUTHORS} \begin{tabular}{l|l} 
Evgeniy Alexandrovich Timofeev & $\begin{array}{l}\text { orcid.org/0000-0002-3094-4390. E-mail: timofeevEA@gmail.com } \\
\text { Sc.D., professor. }\end{array}$
\end{tabular}

For citation: E. A. Timofeev, "On a Segment Partition for Entropy Estimation”, Modeling and analysis of information systems, vol. 27, no. 1, pp. 40-47, 2020. 
THEORY OF COMPUTING

\section{Об одном разбиении отрезка, применяемом для оценки энтропии}

Е. А. Тимофеев ${ }^{1}$

DOI: $10.18255 / 1818-1015-2020-1-40-47$

${ }_{1}^{1}$ Ярославский государственный университет им. П. Г. Демидова, ул. Советская, 14, Ярославль, 150003 Россия.

УДК 519.17

Научная статья

Полный текст на русском языке
Получена 23 ноября 2019 г.

После доработки 18 февраля 2020 г. Принята к публикации 28 февраля 2020 г.

В работе изучается разбиение отрезка, которое строится по следующему правилу:

$$
\begin{aligned}
& Q_{1}=\left\{0, q^{2}, q, 1\right\} . \\
& Q_{n+1}^{\prime}=q Q_{n} \cap q^{2} Q_{n}, \quad Q_{n+1}^{\prime \prime}=q^{2}+q Q_{n} \cap q Q_{n}, \quad Q_{n+1}^{\prime \prime \prime}=q^{2}+q Q_{n} \cap q+q^{2} Q_{n}, \\
& Q_{n+1}=Q_{n+1}^{\prime} \cup Q_{n+1}^{\prime \prime} \cup Q_{n+1}^{\prime \prime \prime},
\end{aligned}
$$

где $q^{2}+q=1$.

Введем последовательность чисел $d=1,2,1,0,1,2,1,0,1,0,1,2,1,0,1,2,1, \ldots$, положив

$$
\begin{aligned}
& d_{1}=1, d_{2}=2, d_{4}=0 ; \\
& d\left[2 F_{2 n}+1: 2 F_{2 n+1}+1\right]=d\left[1: 2 F_{2 n-1}+1\right] \\
& \quad n=0,1,2, \ldots ; \\
& d\left[2 F_{2 n+1}+2: 2 F_{2 n+1}+2 F_{2 n-2}\right]=d\left[2 F_{2 n-1}+2: 2 F_{2 n}\right] \\
& d\left[2 F_{2 n+1}+2 F_{2 n-2}+1: 2 F_{2 n+1}+2 F_{2 n-1}+1\right]=d\left[1: 2 F_{2 n-3}+1\right] \\
& d\left[2 F_{2 n+1}+2 F_{2 n-1}+2: 2 F_{2 n+2}\right]=d\left[2 F_{2 n-1}+2: 2 F_{2 n}\right] \\
& \quad n=1,2,3, \ldots ;
\end{aligned}
$$

где $F_{n}$ - числа Фибоначчи $\left(F_{-1}=0, F_{0}=F_{1}=1\right)$.

Основной результат работы.

теорема.

$$
\begin{gathered}
Q_{n}^{\prime}=1-Q_{n}^{\prime \prime \prime}=\left\{\sum_{i=1}^{k} q^{n+d_{i}}, k=0,1, \ldots, m_{n}\right\}, \\
Q_{n}^{\prime \prime}=1-Q_{n}^{\prime \prime}=\left\{q^{2}+\sum_{i=m_{n}}^{k} q^{n+d_{i}}, k=m_{n}-1, m_{n}, \ldots, m_{n+1}\right\},
\end{gathered}
$$

где $m_{2 n}=2 F_{2 n-2}, m_{2 n+1}=2 F_{2 n-1}+1$.

Ключевые слова: мера; метрика; энтропия; оценка; несмещенность; самоподобие; мера Бернулли

\section{ИНФОРМАЦИЯ ОБ АВТОРАХ}

Евгений Александрович Тимофеев

orcid.org/0000-0002-3094-4390. E-mail: timofeevEA@gmail.com

доктор. физ.-мат. наук., профессор кафедры теоретической информатики.

Для цитирования: E. A. Timofeev, “On a Segment Partition for Entropy Estimation”, Modeling and analysis of information systems, vol. 27, no. 1, pp. 40-47, 2020.

() Тимофеев Е. А., 2020

Эта статья открытого доступа под лицензией CC BY-NC-ND license (http://creativecommons.org/licenses/by-nc-nd/4.0/). 
В работе [1] для обоснования несмещенности оценки энтропии применялась последовательность разбиений отрезка, которая строилась по следующим рекуррентным правилам:

$$
\begin{aligned}
& Q_{1}=\left\{0, q^{2}, q, 1\right\} . \\
& Q_{n+1}^{\prime}=q Q_{n} \cap q^{2} Q_{n}, \quad Q_{n+1}^{\prime \prime}=q^{2}+q Q_{n} \cap q Q_{n}, \quad Q_{n+1}^{\prime \prime \prime}=q^{2}+q Q_{n} \cap q+q^{2} Q_{n}, \\
& Q_{n+1}=Q_{n+1}^{\prime} \cup Q_{n+1}^{\prime \prime} \cup Q_{n+1}^{\prime \prime \prime},
\end{aligned}
$$

где $q^{2}+q=1$.

В настоящей работе будет показано, что $Q_{n}$ - измельчающая последовательность разбиений и отрезки разбиения $Q_{n}$ имеют длины $q^{n}, q^{n+1}, q^{n+2}$. Найдено рекуррентное задание длин отрезков разбиения $Q_{n}$.

Введем последовательность чисел $d=1,2,1,0,1,2,1,0,1,0,1,2,1,0,1,2,1, \ldots$, положив

$$
\begin{aligned}
& d_{1}=1, d_{2}=2, d_{4}=0 ; \\
& d\left[2 F_{2 n}+1: 2 F_{2 n+1}+1\right]=d\left[1: 2 F_{2 n-1}+1\right] ; \\
& \quad n=0,1,2, \ldots ; \\
& d\left[2 F_{2 n+1}+2: 2 F_{2 n+1}+2 F_{2 n-2}\right]=d\left[2 F_{2 n-1}+2: 2 F_{2 n}\right] ; \\
& d\left[2 F_{2 n+1}+2 F_{2 n-2}+1: 2 F_{2 n+1}+2 F_{2 n-1}+1\right]=d\left[1: 2 F_{2 n-3}+1\right] ; \\
& d\left[2 F_{2 n+1}+2 F_{2 n-1}+2: 2 F_{2 n+2}\right]=d\left[2 F_{2 n-1}+2: 2 F_{2 n}\right] ; \\
& \quad n=1,2,3, \ldots ;
\end{aligned}
$$

где $F_{n}$ - числа Фибоначчи $\left(F_{-1}=0, F_{0}=F_{1}=1\right)$.

Теорема 1. Пусть последовательность д задана в (2), тогда

$$
\begin{gathered}
Q_{n}^{\prime}=1-Q_{n}^{\prime \prime \prime}=\left\{\sum_{i=1}^{k} q^{n+d_{i}}, k=0,1, \ldots, m_{n}\right\}, \\
Q_{n}^{\prime \prime}=1-Q_{n}^{\prime \prime}=\left\{q^{2}+\sum_{i=m_{n}}^{k} q^{n+d_{i}}, k=m_{n}-1, m_{n}, \ldots, m_{n+1}\right\},
\end{gathered}
$$

где $m_{2 n}=2 F_{2 n-2}, m_{2 n+1}=2 F_{2 n-1}+1$.

Замечание 1. Последовательность d приведена и в «Энциклопедии целочисленных последовательностей» ${ }^{1}$ под номером А191329, где она задана формулой

$$
d_{n}=[n q+n] \bmod 2+[n q] \bmod 2 .
$$

Замечание 2. Из (3) получаем формулу

$$
q^{2-n}=\sum_{i=1}^{m_{n}} q^{d_{i}}
$$

Доказательство. Для упрощения задания множеств $Q_{n}$ докажем несколько вспомогательных лемм

\footnotetext{
${ }^{1}$ https://oeis.org/
} 
Лемма 1. $Q_{n}^{\prime}=1-Q_{n}^{\prime \prime \prime}, Q_{n}^{\prime \prime}=1-Q_{n}^{\prime \prime}$.

Доказательство. Проведем индукцию по $n$.

При $n=1$ условия леммы выполнены.

Предположим, что они выполнены для $n$ и покажем, что они выполнены при $n+1$.

Из предположений следует, что $Q_{n}=1-Q_{n}$.

Из определения разбиения (1) следует, что

$$
\begin{gathered}
1-Q_{n+1}^{\prime \prime \prime}=q-q Q_{n} \cap q^{2}-q^{2} Q_{n}=q Q_{n} \cap q^{2} Q_{n}=Q_{n+1}^{\prime} . \\
1-Q_{n+1}^{\prime \prime}=q-q Q_{n} \cap 1-q Q_{n}=q\left(1-Q_{n}\right) \cap q^{2}+q\left(1-Q_{n}\right)=q Q_{n} \cap q^{2}+q Q_{n}=Q_{n+1}^{\prime \prime} .
\end{gathered}
$$

Лемма 2. Справедливы соотношения

$$
\begin{gathered}
Q_{n}^{\prime}=q Q_{n-1}^{\prime} \cup q Q_{n-1}^{\prime \prime} . \\
Q_{n}^{\prime} \cap q Q_{n}^{\prime}=q Q_{n-1}^{\prime}, \\
Q_{n}^{\prime} \cap q Q_{n}^{\prime \prime}=q Q_{n-1}^{\prime \prime}, \\
Q_{n}^{\prime \prime} \subset q^{2}+q Q_{n}^{\prime}, \\
Q_{n}^{\prime \prime} \subset q Q_{n}^{\prime \prime \prime}, \\
Q_{n-1}^{\prime} \subset Q_{n}^{\prime}, \\
Q_{n-1}^{\prime \prime} \subset Q_{n}^{\prime \prime},
\end{gathered}
$$

Доказательство. Проведем индукцию по $n$.

При $n=2$ из (1) имеем

$$
Q_{2}=\left\{0, q^{3}, q^{2}, q, q+q^{4}, 1\right\},
$$

поэтому условия леммы выполнены.

Предположим, что они выполнены для $n$ и покажем, что они выполнены при $n+1$.

Докажем (5). Из определения разбиения (1) следует, что

$$
Q_{n+1}^{\prime}=\left(q Q_{n}^{\prime} \cup q Q_{n}^{\prime \prime}\right) \cap\left(q^{2} Q_{n}^{\prime} \cup q^{2} Q_{n}^{\prime \prime} \cup q^{2} Q_{n}^{\prime \prime \prime}\right)=q\left[\left(Q_{n}^{\prime} \cap q Q_{n}^{\prime}\right) \cup\left(Q_{n}^{\prime} \cap q Q_{n}^{\prime \prime}\right) \cup\left(Q_{n}^{\prime \prime} \cap q Q_{n}^{\prime \prime \prime}\right)\right] .
$$

Применяя (6), (7), (9), получим

$$
Q_{n+1}^{\prime}=q\left(q Q_{n-1}^{\prime} \cup q Q_{n-1}^{\prime \prime} \cup Q_{n}^{\prime \prime}\right)
$$

Применяя (5), получим

$$
Q_{n+1}^{\prime}=q\left(Q_{n}^{\prime} \cup Q_{n}^{\prime \prime}\right) .
$$

Докажем (6). Из последнего равенства (12) имеем

$$
Q_{n+1}^{\prime} \cap q Q_{n+1}^{\prime}=\left(q Q_{n}^{\prime} \cup q Q_{n}^{\prime \prime}\right) \cap\left(q^{2} Q_{n}^{\prime} \cup q^{2} Q_{n}^{\prime \prime}\right)=q\left[\left(Q_{n}^{\prime} \cap q Q_{n}^{\prime}\right) \cup\left(Q_{n}^{\prime} \cap q Q_{n}^{\prime \prime}\right)\right] .
$$

Применяя (6), (7), (5), получим

$$
Q_{n+1}^{\prime} \cap q Q_{n+1}^{\prime}=q\left(q Q_{n-1}^{\prime} \cup q Q_{n-1}^{\prime \prime}\right)=q Q_{n}^{\prime \prime} .
$$

Докажем (7). Из определения разбиения (1) следует, что

$$
Q_{n+1}^{\prime \prime}=q^{2}+q Q_{n}^{\prime} \cap q Q_{n}^{\prime \prime \prime}
$$


Timofeev E. A.

Отсюда и из (12) следует, что

$$
\begin{aligned}
Q_{n+1}^{\prime} \cap q Q_{n+1}^{\prime \prime}=\left(q Q_{n}^{\prime} \cup q Q_{n}^{\prime \prime}\right) \cap q^{3}+q^{2} Q_{n}^{\prime} \cap q^{2} Q_{n}^{\prime \prime \prime}=q\left[\left(Q_{n}^{\prime} \cup Q_{n}^{\prime \prime}\right) \cap q^{2}+q Q_{n}^{\prime} \cap q Q_{n}^{\prime \prime \prime}\right]= & \\
= & q\left[Q_{n}^{\prime \prime} \cap q^{2}+q Q_{n}^{\prime} \cap q Q_{n}^{\prime \prime \prime}\right] .
\end{aligned}
$$

Применяя (8), (9), получим

$$
Q_{n+1}^{\prime} \cap q Q_{n+1}^{\prime \prime}=q Q_{n}^{\prime \prime} .
$$

Докажем (8). Подставив (12) и (13), получим, что нужно доказать вложение

$$
Q_{n+1}^{\prime \prime}=q^{2}+q Q_{n}^{\prime} \cap q Q_{n}^{\prime \prime \prime} \subset q^{2}+q^{2} Q_{n}^{\prime} \cup q^{2}+q^{2} Q_{n}^{\prime \prime} .
$$

Применяя (5), получим

$$
q^{2}+q\left(q Q_{n-1}^{\prime} \cup q Q_{n-1}^{\prime \prime}\right) \cap q Q_{n}^{\prime \prime \prime} \subset q^{2}+q^{2} Q_{n}^{\prime} \cup q^{2}+q^{2} Q_{n}^{\prime \prime} .
$$

Последнее вложение выполняется по (10), (11).

Докажем (9). Из леммы 1 и (12) следует, что

$$
Q_{n+1}^{\prime \prime \prime}=q^{2}+q Q_{n}^{\prime \prime} \cap q^{2}+q Q_{n}^{\prime \prime \prime} .
$$

Подставив (13), получим, что нужно доказать вложение

$$
Q_{n+1}^{\prime \prime}=q^{2}+q Q_{n}^{\prime} \cap q Q_{n}^{\prime \prime \prime} \subset q^{3}+q^{2} Q_{n}^{\prime \prime} \cup q^{3}+q^{2} Q_{n}^{\prime \prime \prime}=Q_{n+1}^{\prime \prime \prime} .
$$

Для этого достаточно доказать, что

$$
Q_{n}^{\prime \prime \prime} \subset q^{2}+q Q_{n}^{\prime \prime} \cup q^{2}+q Q_{n}^{\prime \prime \prime} .
$$

Из леммы 1 и (5) следует, что

$$
Q_{n}^{\prime \prime \prime}=q^{2}+q Q_{n-1}^{\prime \prime} \cap q^{2}+q Q_{n-1}^{\prime \prime \prime}
$$

Подставляя, получим вложение

$$
q^{2}+q Q_{n-1}^{\prime \prime} \cap q^{2}+q Q_{n-1}^{\prime \prime \prime} \subset q^{2}+q Q_{n}^{\prime \prime} \cup q^{2}+q Q_{n}^{\prime \prime \prime},
$$

которое выполняется по (10), (11).

Докажем (10). Подставив (5), (12), получим, что нужно доказать вложение

$$
Q_{n}^{\prime}=q Q_{n-1}^{\prime} \cup q Q_{n-1}^{\prime \prime} \subset q Q_{n}^{\prime} \cup q Q_{n}^{\prime \prime}=Q_{n+1}^{\prime},
$$

которое выполняется по (10), (11).

Докажем (11). Подставив (13), получим, что нужно доказать вложение

$$
Q_{n}^{\prime \prime} \subset Q_{n+1}^{\prime \prime}=q^{2}+q Q_{n}^{\prime} \cap q Q_{n}^{\prime \prime \prime} .
$$

Применяя (10), (11), получим

$$
Q_{n+1}^{\prime \prime}=q^{2}+q Q_{n}^{\prime} \cap q Q_{n}^{\prime \prime} \supset q^{2}+q Q_{n-1}^{\prime} \cap q Q_{n-1}^{\prime \prime \prime}=Q_{n}^{\prime \prime} .
$$

44 
Введем обозначения.

Пусть $d=d_{1}, d_{2}, \ldots, d_{m}$ - последовательность целых чисел, тогда положим

$$
\begin{gathered}
\bar{d}=d_{m}, d_{m-1}, \ldots, d_{1}, \\
d^{*}=0, d_{3}, d_{4}, \ldots, d_{m}, \\
Q(d)=\left\{\sum_{i=1}^{k} q^{d_{i}}, k=0,1, \ldots, m\right\} .
\end{gathered}
$$

\section{Лемма 3.}

$$
\begin{gathered}
Q_{n}^{\prime}=q^{n} Q\left(d_{n}^{\prime}\right), \\
Q_{n}^{\prime \prime}=q^{2}+q^{n} Q\left(d_{n}^{\prime \prime}\right),
\end{gathered}
$$

где последовательности $d_{n}^{\prime}, d_{n}^{\prime \prime}$ удовлетворяют рекуррентным уравнениям

$$
\begin{gathered}
d_{2 n}^{\prime}=d_{2 n-1}^{\prime} d_{2 n-1}^{\prime \prime}, \quad d_{2 n}^{\prime \prime}=d_{2 n-1}^{\prime}, \\
d_{2 n-1}^{\prime}=d_{2 n-3}^{\prime} d_{2 n-3}^{\prime \prime} d_{2 n-3}^{\prime}, \\
d_{2 n-1}^{\prime *}=d_{2 n-1}^{\prime \prime} d_{2 n-3}^{\prime}, \\
d_{2 n-1}^{\prime \prime}=d_{2 n-3}^{\prime \prime} d_{2 n-5}^{\prime} d_{2 n-3}^{\prime \prime} .
\end{gathered}
$$

Доказательство. Проведем индукцию по $n$.

$$
\begin{gathered}
Q_{3}^{\prime}=q^{3} Q\left(d_{3}^{\prime}\right)=\left\{0, q^{4}, q^{3}, q^{2}\right\}, d_{3}^{\prime}=1,2,1 ; \\
Q_{3}^{\prime \prime}=q^{2}+q^{3} Q\left(d_{3}^{\prime \prime}\right)=\left\{q^{2}, q\right\}, d_{3}^{\prime \prime}=0 ; \\
Q_{4}^{\prime}=q^{4} Q\left(d_{4}^{\prime}\right)=\left\{0, q^{5}, q^{4}, q^{3}, q^{2}\right\}, d_{4}^{\prime}=1,2,1,0 ; \\
Q_{4}^{\prime \prime}=q^{2}+q^{4} Q\left(d_{4}^{\prime \prime}\right)=\left\{q^{2}, q^{2}+q^{5}, q^{2}+q^{4}, q\right\}, d_{4}^{\prime \prime}=1,2,1 ; \\
Q_{5}^{\prime}=q^{5} Q\left(d_{5}^{\prime}\right)=\left\{0, q^{6}, q^{5}, q^{4}, q^{3}, q^{3}+q^{6}, q^{3}+q^{5}, q^{2}\right\}, d_{5}^{\prime}=1,2,1,0,1,2,1 ; \\
Q_{5}^{\prime \prime}=q^{2}+q^{5} Q\left(d_{5}^{\prime \prime}\right)=\left\{q^{2}, q^{2}+q^{5}, q^{2}+q^{4}, q\right\}, d_{5}^{\prime \prime}=0,1,0 ;
\end{gathered}
$$

Поэтому лемма справедлива при $n \leq 5$. Предположим, что утверждения леммы выполняются при некотором $n$, и покажем, что они выполнены для $n+1$.

Применяя (5) и индукционное предположение, получим

$$
Q_{n+1}^{\prime}=q Q_{n}^{\prime} \cup q Q_{n}^{\prime \prime}=q^{n+1} Q\left(d_{n}^{\prime}\right) \cup q^{3}+q^{n+1} Q\left(d_{n}^{\prime \prime}\right)=q^{2 n+1} Q\left(d_{n}^{\prime} d_{n}^{\prime \prime}\right) .
$$

Следовательно,

$$
d_{n+1}^{\prime}=d_{n}^{\prime} d_{n}^{\prime \prime}
$$

При нечетном $n$ получаем первое равенство в (20). При четном $n$, подставляя (20), получим первое равенство в (21).

Из (13) имеем

$$
Q_{2 n+1}^{\prime \prime}=q^{2}+q^{2 n+2} Q\left(d_{2 n}^{\prime}\right) \cap q^{2}+q^{2 n+2} Q\left({\overline{d^{\prime}}}_{2 n}\right)=q^{2}+q^{2 n+2}\left[Q\left(d_{2 n}^{\prime}\right) \cap Q\left({\overline{d^{\prime}}}_{2 n}\right)\right] .
$$

Найдем общую часть последовательностей $d_{2 n}^{\prime}=d_{2 n-1}^{\prime} d_{2 n-1}^{\prime \prime}$ и ${\overline{d^{\prime}}}_{2 n}=d_{2 n-1}^{\prime \prime}{\overline{d^{\prime}}}_{2 n-1}$.

Из второго равенства в (21) имеем

$$
d_{2 n-1}^{\prime *} d_{2 n-1}^{\prime \prime}=d_{2 n-1}^{\prime \prime} d_{2 n-3}^{\prime} d_{2 n-1}^{\prime \prime}
$$


Поэтому,

$$
d_{2 n}^{\prime}=1,2, d_{2 n-1}^{\prime \prime} d_{2 n-3}^{\prime} d_{2 n-1}^{\prime \prime},{\overline{d^{\prime}}}_{2 n}=d_{2 n-1}^{\prime \prime} d_{2 n-3}^{\prime} d_{2 n-1}^{\prime \prime}, 2,1 .
$$

Поскольку $q+q^{2}=1$,

$$
Q\left(d_{2 n}^{\prime}\right) \cap Q\left({\overline{d^{\prime}}}_{2 n}\right)=Q\left(d_{2 n-1}^{\prime *} d_{2 n-1}^{\prime \prime}\right)
$$

Следовательно,

$$
d_{2 n+1}^{\prime \prime}=d_{2 n-1}^{\prime \prime} d_{2 n-3}^{\prime} d_{2 n-1}^{\prime \prime}
$$

и третье равенство в (21) выполнено.

Подставляя полученное равенство в

$$
d_{2 n+1}^{* *}=d_{2 n}^{* *} d_{2 n}^{\prime \prime}=d_{2 n-1}^{\prime *} d_{2 n-}^{\prime \prime} d_{2 n-1}^{\prime}=d_{2 n-1}^{\prime \prime} d_{2 n-3}^{\prime \prime} d_{2 n-1}^{\prime \prime} d_{2 n-1}^{\prime},
$$

получим второе равенство в (21).

Докажем второе равенство в (20).

Из (13) имеем

$$
Q_{2 n+2}^{\prime \prime}=q^{2}+q^{2 n+3} Q\left(d_{2 n+1}^{\prime}\right) \cap q^{2}+q^{2 n+3} Q\left({\overline{d^{\prime}}}_{2 n+1}\right)=q^{2}+q^{2 n+3}\left[Q\left(d_{2 n+1}^{\prime}\right) \cap Q\left({\overline{d^{\prime}}}_{2 n+1}\right)\right] .
$$

Поскольку

$$
d_{2 n+1}^{\prime}=d_{2 n-1}^{\prime} d_{2 n-}^{\prime \prime} d_{2 n-1}^{\prime}={\overline{d^{\prime}}}_{2 n+1}
$$

получаем

$$
d_{2 n+2}^{\prime \prime}=d_{2 n+1}^{\prime} \text {. }
$$

Найдем величины $m_{n}=\left|d_{n}^{\prime}\right|$.

Из (22) имеем

$$
\left|d_{n}^{\prime \prime}\right|=m_{n+1}-m_{n} .
$$

Подставляя во второе уравнение (21), получим

$$
m_{2 n}=m_{2 n+1}-m_{2 n-1} .
$$

Подставляя (23) в первое уравнение (20), получим уравнение, равносильное (24).

Подставляя (23), (24) в третье уравнение (20), получим рекуррентное уравнение

$$
m_{2 n+1}-4 m_{2 n-1}+4 m_{2 n-3}-m_{2 n-5}=0
$$

с начальным условием $m_{1}=1, m_{3}=3, m_{5}=7$.

Корни характеристического уравнения равны $1, q^{2}, q^{-2}$, их степени выражаются через числа Фибоначчи, поэтому общее решение уравнения (25) имеет вид

$$
m_{2 n+1}=C_{0}+C_{1} F_{2 n-1}+C_{2} F_{2 n-2} .
$$

Найдя константы из начальных условий, получим

$$
m_{2 n+1}=2 F_{2 n-1}+1 \text {. }
$$

Подставляя в(24), получим

$$
m_{2 n}=2 F_{2 n-2}
$$




\section{References}

[1] E. Timofeev, "Existence of an unbiased consistent entropy estimator for the special Bernoulli measure", Modeling and Analysis of Information Systems, vol. 26, no. 2, pp. 267-278, 2019. 